\title{
Multi-agent semantic interoperability in complex energy systems simulation and decision support
}

\author{
Gabriel Santos, Tiago Pinto \\ GECAD \\ Polytechnic of Porto \\ Porto, Portugal \\ \{gajls, tcp\}@isep.ipp.pt
}

\author{
Zita Vale \\ Polytechnic of Porto \\ Porto, Portugal \\ zav@isep.ipp.pt
}

\author{
Juan Manuel Corchado \\ BISITE \\ University of Salamanca (USAL) \\ Salamanca, Spain \\ corchado@usal.es
}

\begin{abstract}
This work introduces a multi-agent systems' society, in power and energy, for simulation and decision support. A semantic data model is considered to describe the domain concepts, providing a way to achieve semantic communication between the systems involved. Sharing a common vocabulary enables systems' interoperability. A semantic registration and search platform, where systems can register their services and/or search for services they require, is also considered to facilitate interoperability in a (semi-)automatic way. Advanced context-aware decision support models and methods based on a strong artificial intelligence approach are also available to enhance players' outcomes from their participation in the sector. The case study presents an integrated laboratorial simulation and emulation infrastructure, enabling a realistic test and validation of the results.
\end{abstract}

Index Terms-- Decision Support System, Energy Resources Management, Multi-agent Simulation, Power and Energy Systems, Semantic Interoperability.

\section{INTRODUCTION}

In the last pair of decades, the electricity sector has suffered major changes trying to make the sector fairer and avoiding the monopolies run by powerful utilities [1], [2]. The well-known European Union (EU) "20-20-20" targets are highly stirring those changes [3]. The EU aims to reach $20 \%$ of energy consumption from renewable-based generation (RBG) by 2020, doubling the $9.8 \%$ value reached in 2010 .

The most significant changes worldwide are the increased penetration of RBG, leading to the adoption of the smart grids (SG) paradigm [4], [5] and the introduction of a competitive approach in the wholesale electricity markets (EM). More recently some aspects of the retail markets have also been subject to changes [2]. SGs quickly evolved from a concept widely accepted by the involved parties to an industrial reality [6]-[8]. The EM restructuring is another major concern of the EU, particularly the formation of a Pan-European EM [9]. An important step in this direction was taken in early 2015, when

The present work has been developed under the MAS-Society Project cofunded by Portugal 2020 "Fundo Europeu de Desenvolvimento Regional" (FEDER) through PO CI, UID/EEA/00760/2013, and PhD grant SFRH/BD/118487/2016. several European spot EMs were coupled on a common daily market platform.

In this context, it becomes essential to the sector's entities to study and analyze the new market mechanisms and their relationships, being able to take the best outcomes out of the different markets. To this end, multi-agent systems (MAS) are particularly suitable to model, study, and analyze complex interactions of dynamic and constantly evolving systems, such as the power and energy systems (PES) sector [10]. MAS must cope with EM's constant evolution, namely with the inclusion and/or update of new market models and constraints, with the complex relationships among players, and with the constants technological and business developments [11]. The smooth inclusion of new business and data models, market mechanisms, types of players, and types of interactions, are some of the key advantages of using MAS [12]-[14].

In this domain, dome reference modelling tools have emerged, such as: AMES, the Agent-based Modelling of Electricity Systems [15]; EMCAS, the Electricity Market Complex Adaptive System [16]; MASCEM, the Multi-Agent Simulator of Competitive Electricity Markets [17]; and MASGriP, the Multi-Agent Smart Grid simulation Platform [18]. These relevant tools are directed to the market regulators and operators perspective, not providing proper decision support to EM players. To overcome this limitation, the Adaptive Decision Support for Electricity Market Negotiations (AiD-EM) [19] has been developed, aiming at supporting market players in their negotiation process.

Despite relevant advances made in the areas of simulation and decision support in EM and SG, there is yet a generalized limitation related with the lack of interoperability amongst heterogeneous MAS. With interoperable MAS, in PES scope, the problem could be addressed as a whole, from EM to the end consumer, considering all the relevant existing business models and stakeholders' interrelationships. This is especially relevant when considering the introduction of small players in EM's paradigm (e.g. players of local EM in the frame of SG). Such interoperability would benefit a strong improvement in EM and SG studies and development.

Ontologies facilitate the interoperability between heterogeneous systems giving semantic meaning to information exchanged between heterogeneous parties [20]. In PES, there are several proposals for the use of ontologies [21]-[25], with emphasis on IBM's ontology developed under 
the Insights Foundation for Energy Data Model [26] and also in EM field [27]-[29]. Some European projects are also advancing in the reasoning on ontologies, namely the ENERGy [30] and Web of Objects [31] projects. However, most of these ontologies are focused on very specific utilities' needs, while others, such as [27], only consider a high-level domain ontology, leaving aside the application scenario, hardening their use in real world use cases. Moreover, most of these ontologies are not publicly available, which hinders their reuse and extension.

It is thus essential to develop and make available ontologies that allow the representation of different, but transversal, knowledge sources, aiming to facilitate interactions between entities of heterogeneous domains, while enabling systems to leverage semantic reasoning for validating business rules, contracts, among others. In [32] is addressed a brief motivation for the necessity of developing ontologies as a solution to enable heterogeneous Multi-Agent Systems (MAS) to interoperate. The discussion culminates with the expected advantages of creating a so-called Multi-Agent Systems Society, where heterogeneous systems interoperate using semantic communications, enabling also the participation of external systems that may emerge in the future.

This work presents a MAS Society designed and developed to enable the integration of distinct power and energy MAS, allowing the simulation of scenarios involving stakeholders from the end consumer, through the aggregators' resources management, to operators and participants of the wholesale EM. This way it allows to perform joint simulations between several systems, allowing for a more in-depth study, as well as allowing the management of multiple sources of knowledge from heterogeneous natures, by integrating systems with heterogeneous data sources in a common vocabulary.

After the introductory section, section II provides an overview on the proposed MAS Society. Section III overviews semantic interoperability, introducing the ontologies used in the MAS Society. A case study based on real data is featured in section IV. Section V discloses the main conclusions and future work.

\section{MAS SOCIETY}

As discussed in the Introduction, there are multiple approaches for the study and simulation of specific problems, but there aren't currently significant initiatives that allow putting those pieces together in order to enable the study of the global problem, including the interaction between stakeholders and systems of different dimensions and roles. The development of approaches that support this kind of interoperability is essential to allow testing new business, market and players' models and the respective methods and tools supporting their effective application to enable players to actively contribute to the efficient use of the available energy resources.

To this end, this work proposes the integration of several independent MAS, directed to the study of distinct areas of the PES, as a MAS Society. It includes several distinct and independent MAS, covering the entire energy system from wholesale EM simulation to the end users' consumption. It enables the modelling of the system, as a whole, through the interoperation between the involved entities; as well as only partially with the parts desired for the study case in hands. The MAS Society addresses the lack of appropriate means to enable the effective interoperability between different systems, algorithms and methods in PES.

MASCEM [17] is the EM simulator. It provides a diversity of EM models, from three different European markets, namely: MIBEL, EPEX and NordPool; while allowing the simulation of combinations of different models of those markets. MASCEM agents include the system and market operators, the buyer and seller players, and the aggregators. Through semantic interoperability, it also enables the participation of external players that wish to participate in the wholesale EM negotiations, such as SG operators and aggregators. MASGRiP [18] simulator, in turn, provides the modelling of local players at the SG and microgrid level. It enables the management and control of the most relevant stakeholders acting in this environment. The system considers both fully simulated agents, as well as software agents controlling real hardware. This way, the system provides the means to test alternative complex approaches in a realistic setting [33]. AiD-EM [19] providences decision support to EM negotiations. It includes agents to execute different tasks, such as decision support for auction-based markets, and bilateral contracts, and the EM participation portfolio optimization.

The Intelligent Decision Support Multi-Agent System (IDeSMAS) provides the several agents in the community with forecast, scheduling, demand response (DR) programs algorithms, among others. The control of physical devices is achieved through another agent-based tool, the Programmable Logic Controller Multi-Agent System (PLCMAS). It enables to read data from real hardware (e.g.: lights, sockets, airconditioners, sensors, etc.) at a given timestep, and control some of them, being able to test scenarios in a real environment, applying the results in real devices, making them act accordingly. However, those devices must be connected to a Programmable Logic Controller (PLC). Finally, to control the simulations in the MAS Society, the Tools Control Centre (TooCC) is used [34]. It allows the co-simulation of all or some systems/algorithms included in the agents' society, and also the simulation of each system independently. The automatic analysis of the various sources of knowledge and simulations' results is facilitated in an integrated manner.

The MAS Society allows to model the PES as a whole, representing the most relevant actors through software agents, in the respective MAS. The main advantage is that it allows the study and simulation of diverse and complex scenarios involving one or more systems dedicated to distinct problems. Using TooCC, different complex dynamics between different MAS agents can be realized and customized, configured and analyzed. All MAS composing the MAS Society are developed using the Java Agent DEvelopment (JADE) framework, making the system compliant with the specifications of the Foundation for Intelligent Physical Agents (FIPA). FIPA specifications also define a set of guidelines to achieve agents' interoperability. However, these are not enough to ensure that agents are able to communicate 
efficiently and without misinterpretations. The following section presents a complementary solution to fulfill the required interoperability needs.

\section{SEMANTIC INTEROPERABILITY}

To reach the required interoperability, agents must share a common vocabulary in a given context, to disambiguate similar concepts with different meanings. To this end, this paper advocates the use of ontologies, which allow vocabulary sharing and concept mapping between systems, so that they can communicate effectively and without misunderstandings. Ontologies allow heterogeneous systems to speak the same language and to comprehend the same concepts and terms, preventing different interpretations on the exchanged information.

This work uses two types of ontologies. One is the conceptual type which represents transversal concepts that are the basis for systems' communication. These ontologies describe the vocabulary shared among systems. The other is the applicational type, which is related with the procedures of each system. These latter describe the systems' services and communications, detailing their respective inputs and outputs. The ontologies developed are publicly available so they may be extended and reused to enable full interoperability between simulation tools of the PES. They provide the means to successfully share meaningful data that is easily interpreted by agent-based systems. The following subsections introduce the ontologies used, extended and developed for this work.

\section{A. Electricity Markets Ontology}

The Electricity Markets Ontology (EMO) [35], [36] has been developed to describe abstract concepts and axioms in the EM domain from the wholesale to local markets. It aims to be as comprehensive as possible so it can be extended and reused in the development of market-specific ontologies, independently of the market's rules and features. EMO is the base ontology from which several lower-level ontologies have been extended, as is the case of MIBEL (MBL) [37], EPEX (EPX) [38], NordPool (NPO) [39] and AiD-EM (ADM) [40] ontologies. EMO also considers two additional modules to facilitate semantic communications between player and market operator agents [41], namely: Call For Proposal (CFP) and Electricity Markets Results (EMR) ontologies. CFP and EMR are independent from EMO as they do not directly integrate concepts that define an EM; rather concepts that are related to an EM. The CFP is used by the market operator agents to request player agents to bid on the market, and for players to send their bids to the respective market operators. In turn, the EMR ontology is used by market operator agents to notify player agents about their market results and outcomes.

The MBL [37] ontology represents concepts and properties that describe the Iberian EM. It is an extension of the EMO while including some new concepts and relations specific of the Iberian market. The EPX [38] and NPO [39] ontologies are very similar due to the similarity of the EM they represent. They have been developed to describe concepts and properties from EPEX Spot and NordPool Spot EM, respectively. Both ontologies import and extend EMO's concepts and relations, adding the necessary terms and properties to describe each EM. Finally, the ADM [40] ontology has been developed aiming at providing AiD-EM's interoperability with any EM player of any agent-based simulation platform, providing any EM player with decision support. It also imports EMO's concepts and extends it by adding new classes, object properties and data properties; and relating them with EMO's players.

The EMO ontology, and respective extensions, have been developed to provide MASCEM and AiD-EM agents with semantic interoperability. These ontologies were formulated in OWL DL, using Protégé ${ }^{2}$ tool, and its representation is in RDF/XML. They are publicly available ${ }^{3}$ for third party developers wishing to integrate their tools with MASCEM leveraging from its market models and simulation capabilities. EMO may also be extended and reused as needed in the development of agent-based simulators. More details about each ontology module are available in the respective references.

\section{B. Smart Energy Aware Systems ontology}

The Smart Energy Aware Systems (SEAS) [42] ontology has been designed to describe the SEAS project ${ }^{4}$ knowledge model as basis for the semantic interoperability in the project's ecosystem. SEAS project solves the inefficient energy consumption problem, which results from a lack of enough means to control, monitor, estimate, and adapt systems' energy usage dynamically in response to the environment in context. The main goal of the project was to design and develop an ecosystem of IoT services and smart devices capable of ensuring future stability and efficiency of the power grid.

SEAS ontology has been designed to meet the best practices relatively to quality, metadata, and publication; to reuse or align to existing standards; to cover the SEAS project use cases expressivity; and to be extensible to other use cases. The SEAS knowledge model, together with the SEAS architecture, allows to expose, exchange, reason and query knowledge in a semantically interoperable manner consisting of a set of OWL2 DL ontology modules [42]. The SEAS ontology modules reuse or align with some existing standards and are publicly available at https://w3id.org/seas.

In this work, the SEAS ontology is used mainly for the MAS Society systems' interoperability with MASGriP, which deals with the microgrid/SG paradigm.

Recently, an effort has been made to integrate SEAS ontology with the Smart Appliances REFerence ontology (SAREF) [43].

\section{Smart Appliances REFerence ontology}

The Smart Appliances REFerence (SAREF) ontology [25] is a shared model designed to make it easier to match existing assets in the smart appliances domain. It provides building blocks that enable separation and recombination of distinct pieces of the ontology depending on the specific needs. It has been developed to gather semantics and data from buildings and households' smart appliances. SAREF allows to combine data from different organizations while fitting into the machine-to-machine (M2M) architecture of the European Telecommunications Standard Institute (ETSI).

\footnotetext{
${ }^{2}$ http://protege.stanford.edu/

${ }^{3} \mathrm{http}: / /$ www.mascem.gecad.isep.ipp.pt/ontologies/

${ }^{4} \mathrm{https}$ ///itea3.org/project/seas.html
} 
The concept Device is SAREF's starting point. SAREF provides a set of basic functions that, when combined, enable more complex functions on a single device. Depending on the device's functions, the device can assume some corresponding states. Devices can offer services, which can represent one or multiple functions. In SAREF ontology, a device can also be described by an Energy/Power profile. This profile may be used to improve the energy efficiency of an office or apartment of a building. SAREF ontology is publicly available at http://ontology.tno.nl/saref.ttl.

In this work, SAREF ontology has been used for the interoperability between SG operators and the end users that have DR contracts, enabling the dynamic cut/shifting of loads.

\section{Intelligent Energy Systems Ontologies}

The Intelligent Energy Systems Ontologies (IESO), in their turn, are application ontologies developed by the authors research group to describe the different optimization, scheduling and forecasting algorithms, in the area of PES, available in IDeSMAS. IESO also includes the PLC ontology, which is used to interoperate with the PLCMAS, which enables the control and measurement of devices and sensors connected to a PLC.

The IESO ontologies provide the means for the remaining MAS Society systems to interoperate with both IDeSMAS and PLCMAS. While IDeSMAS is useful for both operators and players in the field of energy markets, the PLCMAS bridges the gap between the simulated and the realistic case studies scenarios. Finally, IESO is also publicly available ${ }^{5}$.

The presented ontologies not only represent the necessary concepts to comprehend and use empirical data from distinct sources, as they allow semantic interoperability among the MAS Society heterogeneous systems. These data can be acquired from databases, online, or in real time through analyzers/sensors connected to a PLC. These ontologies provide knowledge representation in a common vocabulary, regardless of the source. Aim to achieve an enhanced simulation platform for fully transactive energy systems, ontologies facilitate the interoperability between the several tools, data, and knowledge sources.

\section{CASE STUDY}

The present case study intends to demonstrate the applicability and usefulness of the proposed ontologies to achieve an interoperable MAS Society for the PES simulation and study. For our application scenario, let's consider a MASGriP SG operator agent called Network Manager (NM) that manages a SG where each network node is represented by a Facility Manager (FM) agent. The NM aggregates several small players with DR contracts so it can be able to participate in MASCEM's wholesale EM. To determine the price to bid in the EM, the NM requests AiD-EM's decision support. Regarding the FM agents, attention will be given to 2 specific agents, namely: FM-Home and FM-Office. The first agent is a small player managing a house (simulated in our laboratory), while the second manages an office (our real office building). The remaining FM agents won't be connected to any hardware. It must be stressed that FM-House will interoperate with both IDeSMAS for scheduling and forecast algorithms, and PLCMAS for loads control and monitoring; while FMOffice will be using an external semantic IoT system for building's energy management, called BRICKS. Figure 1 presents the scenario.

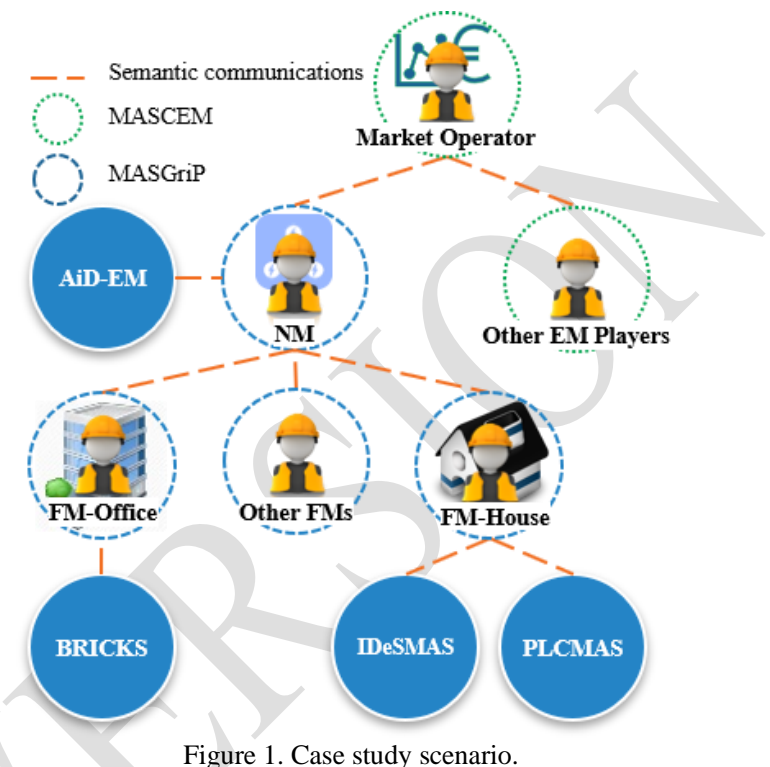

Figure 1. Case study scenario.

The simulation starts with the market operator (MO) sending the call for proposals (CfP) to each player registered in the market. Figure 2 shows a snippet of the CfP sent by the MO, in Turtle ${ }^{6}$ syntax.

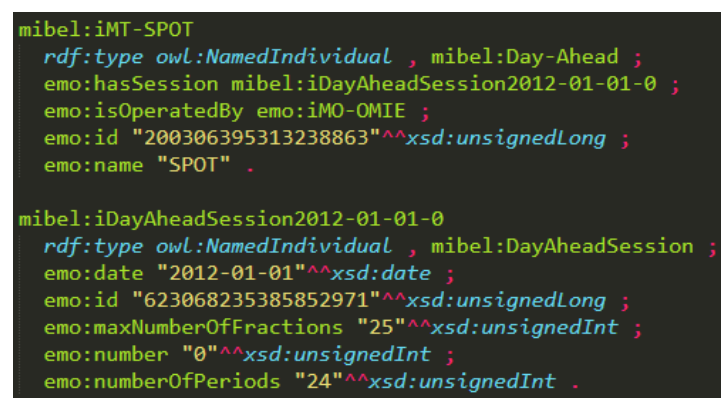

Figure 2. Snippet of the CfP sent by the MO

Observing Figure 2, one can identify the definition of a Day-Ahead pool, named "SPOT", and respective Day-Ahead Session with 24 hourly periods and accepting a maximum of 25 fractions per period bid. Due to size restrictions, only the most relevant snippets will be shown.

Before responding to $\mathrm{MO}$, the $\mathrm{NM}$ requests a consumption forecast, for each hourly period of the next day, to each of his aggregated players. After aggregating the forecasts results, the NM requests AiD-EM decision support to determine the prices for each period bid. Figure 3 presents a snip of the prices suggested by AiD-EM and sent to the MO as response to the CfP. 


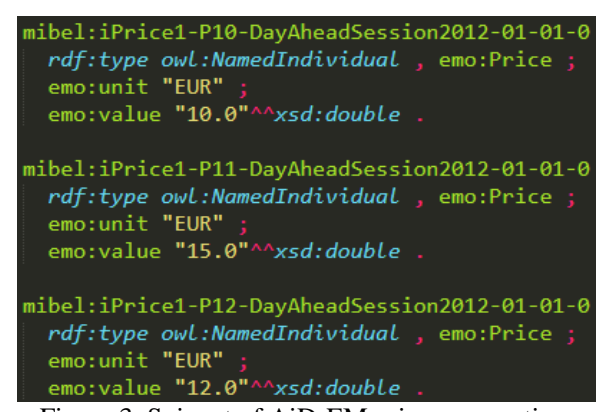

Figure 3. Snippet of AiD-EM prices suggestion.

From Figure 3, the prices suggested for periods 10 to 12 are identifiable. After receiving the prices suggestion, the NM sends his proposal to the MO. The MO executes the EM pool and responds to each player with the results. Figure 4 shows the NM results.

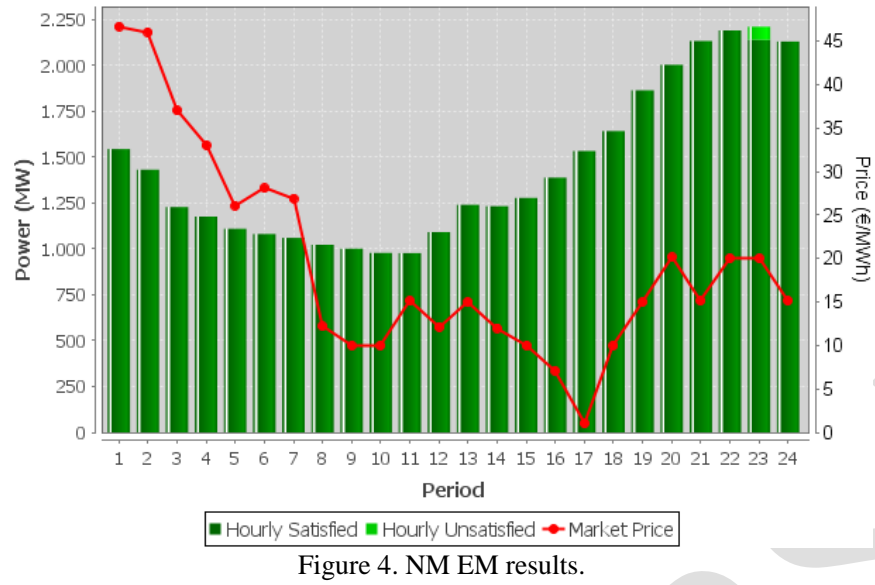

As it can be observed, the NM was able to sell all the available energy in most periods, except in period 23 . However, to make sure he will accomplish the amount of available energy, he may request his aggregated players to reduce some of their consumption through DR events. That is the case of the hourly period 14. Focusing only on players FM-House and FM-Office, the NM requests each to reduce $30 \%$ and $25 \%$, respectively. In the case of FM-Office, the $\mathrm{NM}$ has permission to automatically reduce that percentage through BRICKS system, since they celebrated a contract where FM-Office put available the controllable loads NM can operate. On the other hand, FM-Office runs a scheduling algorithm in IDeSMAS before using PLCMAS to cut/reduce the intended loads. Figure 5 presents a snip of the scheduling result.

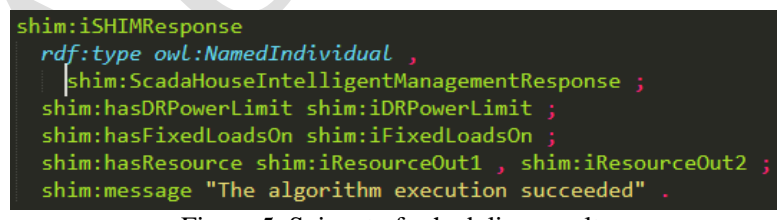

Figure 5. Snippet of scheduling result.

Observing Figure 5 it is possible to verify the DR power limit, and also the considered resources instances, together with a message regarding the algorithm execution success.
In turn, Figure 6 shows a snippet of the request sent by FM-House to PLCMAS to cut/reduce the respective loads.

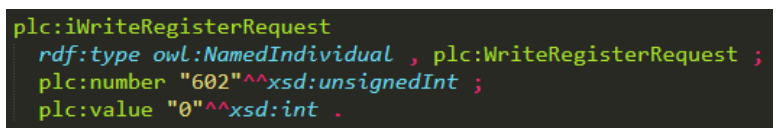

Figure 6. Snip of cut/reduce request.

In the snippet of Figure 6 is possible the see the request to cut a load within the PLC, with register number 602 . For such, 0 (zero) value must be written in register 602 .

Finally, both FM-House and FM-Office agents are remunerated accordingly for the amount of energy reduced/cut in DR events.

\section{CONCLUSIONS}

Interoperability issues must be addressed to spread the development of interoperable MAS in the PES area. These systems' interoperability addresses the growing need for knowledge exchange which enables to take full advantage of these simulation tools. To overcome this issue, the use of semantic interoperability is proposed in this work. This work presented a MAS Society that makes use of semantic models to provide interoperability between the different tools. The ontologies that were developed, reused, and extended have also been introduced, while explaining to what systems they were useful to. Using these vocabularies, software agents can communicate in a common language while providing the means for any agent outside the systems to do the same simply by importing and communicating with the semantics introduced.

The presented ontologies are publicly available online so they can be easily accessed, reused and extended, not only to communicate with the MAS Society tools, but also to be useful for newly developed systems in PES scope. This is a relevant contribution, not only in providing participation in joint power and energy simulations, but also in supporting the development of other system-specific ontologies.

The presented case study has shown the usefulness of using the proposed semantic models in PES simulations. The use of the proposed ontologies provides the MAS Society with a solid platform for studying and exploring the implications and consequences of new and existing approaches in PES. This type of tool is considered essential by researchers in the area in preparing to handle the everchanging PES environment.

\section{REFERENCES}

M. Shahidehpour, H. Yamin, and Z. Li, "Market Overview in Electric Power Systems," in Market Operations in Electric Power Systems: Forecasting, Scheduling, and Risk Management, New York, USA: John Wiley \& Sons, Inc., 2002, pp. 1-20.

[2] F. P. Sioshansi, Evolution of global electricity markets: new paradigms, new challenges, new approaches. Elsevier Science \& Technology, 2013.

[3] European Commission, "2020 climate \& energy package," 2016.

[4] H. Lund, Renewable Energy Systems, 2nd Editio. Elsevier Inc., 2014.

[5] N. Hatziargyriou, Microgrid: architectures and control. Wiley IEEE, 2014.

[6] S. Borlase, Smart grids : infrastructure, technology, and solutions, 1st Edition. CRC Press, 2013. 
[7] F. Gangale, J. Vasiljevska, C. F. Covrig, A. Mengolini, and G. Fulli, "Smart grid projects outlook 2017: Facts, figures and trends in Europe," 2017.

[8] "The European Technology and Innovation Platform Smart Networks for Energy Transition," 2016.

[9] "PCR report, 'ITALIAN BORDERS SUCCESSFULLY COUPLED," 2015.

[10] F. Lopes and H. Coelho, Eds., Negotiation and Argumentation in Multi-agent Systems: Fundamentals, Theories, Systems and Applications. 2014

[11] H. Morais, P. Kádár, P. Faria, Z. A. Vale, and H. M. Khodr "Optimal scheduling of a renewable micro-grid in an isolated load area using mixed-integer linear programming," Renew. Energy, vol. 35, no. 1, pp. 151-156, 2010.

[12] G. Santos et al., "Multi-agent simulation of competitive electricity markets: Autonomous systems cooperation for European market modeling," Energy Convers. Manag., vol. 99, pp. 387-399, 2015.

[13] G. Santos, R. Fernandes, T. Pinto, I. Praça, Z. Vale, and H. Morais, "MASCEM: EPEX SPOT Day-Ahead market integration and simulation," in Intelligent System Application to Power Systems (ISAP), 2015 18th International Conference on, 2015, pp. 1-5.

[14] G. Santos, T. Pinto, Z. Vale, H. Morais, and I. Praça, "Balancing market integration in MASCEM electricity market simulator," in 2012 IEEE Power and Energy Society General Meeting, 2012, pp. $1-8$.

[15] H. Li and L. Tesfatsion, "Development of Open Source Software for Power Market Research: The AMES Test Bed," Iowa State Univ. Dep. Econ. Staff Gen. Res. Pap., vol. 2, 2009.

[16] V. S. Koritarov, "Real-world market representation with agents," IEEE Power Energy Mag., vol. 2, no. 4, pp. 39-46, 2004.

[17] G. Santos, T. Pinto, I. Praça, and Z. Vale, "MASCEM: Optimizing the performance of a multi-agent system," Energy, vol. 111, pp. 513-524, 2016

[18] P. Oliveira, T. Pinto, H. Morais, and Z. Vale, "MASGriP - A Multi-Agent Smart Grid Simulation Platform," in 2012 IEEE Power and Energy Society General Meeting, 2012, pp. 1-8.

[19] T. Pinto, Z. Vale, I. Praça, J. E. Pires, and F. Lopes, "Decision Support for Energy Contracts Negotiation with Game Theory and Adaptive Learning," Energies, vol. 8, no. 9. 2015.

[20] IEEE PES, "IEEE PES Multi-Agent Systems Working Group," 2010. .

[21] Y. Pradeep, S. A. Khaparde, and R. K. Joshi, "High Level Event Ontology for Multiarea Power System," IEEE Trans. Smart Grid, vol. 3, no. 1, pp. 193-202, Mar. 2012.

[22] E. Dogdu et al., "Ontology-centric data modelling and decision support in smart grid applications a distribution service operator perspective," in 2014 IEEE International Conference on Intelligent Energy and Power Systems (IEPS), 2014, pp. 198-204.

[23] A. Espinoza, M. Ortega, C. Fernandez, J. Garbajosa, and A. Alvarez, "Software-intensive systems interoperability in Smart Grids: A semantic approach," in 2011 9th IEEE International Conference on Industrial Informatics, 2011, pp. 739-744.

[24] F. den Hartog, L. Daniele, and J. Roes, "Toward semantic interoperability of energy using and producing appliances in residential environments," in 2015 12th Аnnual IEEE Consumer Communications and Networking Conference (CCNC), 2015, pp. $170-175$.

[25] L. Daniele, "SAREF: the Smart Appliances REFerence ontology."
2016.

[26] IBM, "The Insights Foundation for Energy Data Model," 2017. .

[27] P. Alexopoulos, K. Kafentzis, and C. Zoumas, ELMO: An Interoperability Ontology for the Electricity Market. 2009.

[28] K. Van Dam and E. Chappin, "Coupling Agent-Based Models of Natural Gas and Electricity Markets," 2019.

[29] K. H. van Dam and J. Keirstead, "Re-use of an ontology for modelling urban energy systems," in Next generation infrastructure systems for eco-cities, 2010, pp. 1-6.

[30] "ITEA 3 · Project · 04024 ENERGy.".

[31] "ITEA 3 - Project - 10028 Web of Objects." .

[32] G. Santos, T. Pinto, and Z. Vale, "Ontologies for the Interoperability of Heterogeneous Multi-agent Systems in the Scope of Power and Energy Systems," in Trends in Cyber-Physical Multi-Agent Systems. The PAAMS Collection - 15th International Conference, PAAMS 2017, 2018, pp. 300-301.

[33] L. Gomes, P. Faria, H. Morais, Z. Vale, and C. Ramos, "Distributed, Agent-Based Intelligent System for Demand Response Program Simulation in Smart Grids," IEEE Intell. Syst., vol. 29, no. 1, pp. 56-65, Jan. 2014.

[34] B. Teixeira et al., "Multi-Agent Decision Support Tool to Enable Interoperability among Heterogeneous Energy Systems," Appl. Sci., vol. 8, no. 3, p. 328, Feb. 2018.

[35] G. Santos, T. Pinto, Z. Vale, I. Praça, and H. Morais, "Electricity Markets Ontology to Support MASCEM's Simulations," in Highlights of Practical Applications of Scalable Multi-Agent Systems. The PAAMS Collection: International Workshops of PAAMS 2016, Sevilla, Spain, June 1-3, 2016. Proceedings, J. Bajo, J. M. Escalona, S. Giroux, P. Hoffa-D kkabrowska, V. Julián, P. Novais, N. Sánchez-Pi, R. Unland, and R. Azambuja-Silveira, Eds. Cham: Springer International Publishing, 2016, pp. 393-404.

[36] G. SANTOS, T. PINTO, Z. VALE, I. PRAÇA, and H. MORAIS, "Enabling Communications in Heterogeneous Multi-Agent Systems: Electricity Markets Ontology," ADCAIJ Adv. Distrib. Comput. Artif. Intell. J., vol. 5, no. 2, 2016.

[37] G. Santos, T. Pinto, I. Praça, and Z. Vale, "Iberian electricity market ontology to enable smart grid market simulation," Energy Informatics, vol. 1, no. 1, p. 13, Sep. 2018.

[38] G. Santos, T. Pinto, I. Praca, and Z. Vale, "EPEX ontology: Enhancing agent-based electricity market simulation," in 2017 19th International Conference on Intelligent System Application to Power Systems (ISAP), 2017, pp. 1-6.

[39] G. Santos, T. Pinto, I. Praça, and Z. Vale, "Nord Pool Ontology to Enhance Electricity Markets Simulation in MASCEM," in Progress in Artificial Intelligence, 2017, pp. 283-294.

[40] G. Santos, "Ontologies for the interoperability of multiagent electricity markets simulation platforms," Institute of Engineering Polytechnic of Porto , 2015.

[41] G. Santos, T. Pinto, I. Praça, and Z. Vale, "An Interoperable Approach for Energy Systems Simulation: Electricity Market Participation Ontologies," Energies, vol. 9, no. 11, 2016.

[42] M. Lefrançois, J. Kalaoja, T. Ghariani, and A. Zimmermann, "SEAS Knowledge Model," 2016.

[43] M. Lefrançois, "Planned ETSI SAREF extensions based on the W3C\&OGC SOSA/SSN-compatible SEAS ontology patterns," in CEUR Workshop Proceedings, 2017, vol. 2063, p. 11p. 